\title{
Pancreatoduodenectomy due to cancer in the older population
}

\author{
Jakub Kenig, Piotr Richter \\ Department of General, Gastrointestinal, Oncologic Surgery and Transplantology, I Chair of General Surgery, \\ Jagiellonian University Medical College, Krakow, Poland
}

By $2030,70 \%$ of all pancreatic cancer will be diagnosed in the older population. However, pancreas operations are a complex surgical procedure with a high rate of morbidity and mortality. Therefore, the benefits of surgical resection in older patients are controversial and decisions about treatment for this group must be well balanced. Chronological age alone should not be a contraindication for multimodal radical treatment in older patients. Fit patients, according to the comprehensive geriatric assessment (SGA), should be qualified for the same treatment as younger patients to benefit the same outcomes. However, they should be operated on in high-volume hospitals by experienced surgeons. Prefrail patients should undergo prehabilitation, during neoadjuvant treatment also, and then reevaluated. Frail patients should be discussed in an oncogeriatric meeting. We still do not have evidence-based data to design a tailored approach for them so as to balanced good oncologic outcomes and the appropriate postoperative quality of life.

Key words: older patients, pancreatic cancer, pancreatoduodenectomy, elderly

Pancreatic ductal adenocarcinoma (PC) is common in the older population, with incidence increasing with age, reaching the highest peak after 60 years of age. It is estimated that by 2030, approximately $70 \%$ of PC will be diagnosed in this group [1]. It also has one of the worst prognoses of all malignancies. At present, 5-year relative survival is 8\% [2] and improvements seen for most cancers over the last 20 years, is unfortunately not the case for $\mathrm{PC}$ and progress remains very slow. Surgical resection is the only curative treatment option; it is possible in only $15-20 \%$ of patients. Even among those who undergo surgery, 5-year survival is just 20-25\% due to local or metastatic relapse during the first two years after resection. Thus, PC has the potential to become the second leading cause of cancer-related death before 2030 [3].

Resection of the pancreas (pancreaticoduodenectomy, partial or total pancreatectomy) is a complex surgical procedure with a high rate of morbidity and mortality. Compared with younger patients, many older patients may not be good candidates for surgery, and they are less likely to receive other treatments. Therefore, the benefits of surgical resection for $\mathrm{PC}$ in older patients remain controversial and the decision about this treatment in older patients must be well balanced. Moreover, the most important problem in the treatment of older patients with PC is the underrepresentation of this population in clinical trials. This results in treatment decisions taken for older patients that are extrapolated from studies performed on younger patients. Although the situation is improving constantly, most of the studies still use chronological age, the Karnofsky scale or the Eastern Cooperative Oncology Group/World Health Organization scale and not biological age [4].

\section{How to cite:}

Kenig J, Richter P. Pancreatoduodenectomy due to cancer in the older population. NOWOTWORY J Oncol 2021; 71: 321-327.

This article is available in open access under Creative Common Attribution-Non-Commercial-No Derivatives 4.0 International (CC BY-NC-ND 4.0) license, allowing to download articles and share them with others as long as they credit the authors and the publisher, but without permission to change them in any way or use them commercially. 


\section{Clinical and pathological characteristics of PC in older patients}

Very little data is available regarding PC in the older population. Kamisawa et al. compared the pathologic features of it in older and younger patients and found no differences in location, stage, grade and local spread, although older patients seem to develop fewer hematogenous metastases. Older patients may present more diploid tumours or more p53 mutations, which are associated with a poorer prognosis [5].

\section{Preoperative assessment and treatment decisions}

As was mentioned in our previous publications, the population of older patients is very heterogeneous in terms of co-morbidity, physical reserve, cognitive function and social support. Chronological age alone is a poor predictor of cancer treatment outcomes and toxicities [6]. Current routine pre-operative assessment cannot adequately identify patients at risk. Many older adults have unidentified, uncommunicated, and therefore unaddressed aging-related conditions that are associated with morbidity and early mortality. Therefore, the Comprehensive Geriatric Assessment (CGA) was introduced to help determine the primary status of the older patient, to diagnose frailty syndrome and to identify how to optimise the patient's condition before the start of treatment [7-10].

Frailty syndrome (a surrogate of biological age), is defined as a multisystem reduction in reserve capacity leading to shorter life expectancy, higher risk of complications after surgery/chemotherapy, higher risk of hospital readmission and institutionalisation. Considering their limited remaining lifetime and their postoperative quality of life, the CGA is as valuable as the need to cure or remove their cancers. Therefore, the International Society of Geriatric Oncology (SIOG) and The European Society for Medical Oncology (ESMO) recommends the use of the CGA to determine the biological age before the beginning of treatment. Rostoft et al. analysed the literature regarding the role of the CGA in predicting the outcome in hepatobiliary and pancreatic surgery among older patients with cancer; they concluded that although scarcely investigated, frailty and elements from the CGA are significantly associated with negative short- and long-term treatment outcomes in older patients with HBP [11].

In general, based on the CGA, we can differentiate three groups of older patients:

1. Fit: patients without any deficits in the CGA domains. In this group, standard oncologic treatment can be offered and postoperative outcomes are comparable to younger patients.

2. Pre-frail: patients with one or two deficits in the CGA domains. In these patients prehabilitation should be recommended to improved resilience to surgical stress by, at least, augmenting functional capacity and nutritional status before surgery.
3. Frail patients: patients with three or more impaired domains in the CGA. A tailored approach should be discussed in a geriatric multidisciplinary team meeting [9].

It is also possible to determine the severity of the frailty using the cumulative deficit model for the CGA [10]. Such assessments may guide treatment decisions through evaluations of the balance of benefits and risk-factors associated with performing or omitting specific oncologic interventions.

\section{To operate or to not operate?}

There have been only a few studies that compare the prognosis of patients with resectable pancreatic cancer between the surgery and no surgery group. He et al. showed that the first group had a significantly higher 5-year OS rate (25.0 vs. 2.3\%; $p<0.0001$ ) and a higher median survival time (24.3 vs. 5.8 months) [12]. Similarly, in the study by Park HM et al., surgical resection resulted in better prognosis than the non-surgical approach. Only for patients with a high Charlson comorbidity index was this approach not beneficial [13]. In turn, Marmor et al. reported that for the overall cohort, the median survival rate was significantly longer for patients treated with pancreatectomy as compared with chemotherapy (15 months vs. 10 months). However, for patients 80 years of age and older, the absolute survival benefit was only 3 months (13 months vs. 10 months). Similarly, for patients who underwent pancreatectomy and had positive lymph nodes, the median survival benefit was only 3 months compared to chemotherapy (13 months vs. 10 months) [14]. None of the studies investigated any elements of the CGA.

To conclude, fit and pre-frail patients based on the CGA, should be operated on (the latter group after prehabilitation) with no regard for the chronological age. We do not have good data to draw a conclusion about what would be most beneficial for frail patients in the long-term follow-up. In severe frailty, the best support treatment seems the best option.

\section{It surgery safe for older patients?}

The most up-dated systematic review and metanalysis on pancreatoduodenectomy in older patients was performed by Ten E. et al. in 2019. The study included 12 retrospective studies with 4860 patients. There were 919 patients in the older group and 3941 patients in the younger group. The authors concluded that pancreatic surgery had become a safe procedure for older patients in high-volume hospitals when operated on by an experienced surgeon [15].

The general postoperative mortality rate decreased from $30 \%$ in the 1980 s to $1 \%$ at the present time. However, the complication rate remains high at 40-70\%. A similar situation is reported in older patients, it does not matter what kind of age cut-off was used to define "elderly". In comparison to the younger population, some authors report higher postoperative morbidity and mortality, a higher requirement for an intensive care unit stay, increased length of hospital 
stay and higher rates of hospital readmission after pancreatectomy [16-20]. There are also studies reporting lack of significant differences between these groups. The reason for the significant differences was the volume of the hospital; $<50$ vs. $>50$ pancreatoduodenectomies per year. Across high volume centres, there was no significant difference in the rate of overall and major complications between patients $\geq 80$ years old and $<80$ years. Higher volume centres also had significantly lower in-hospital mortality and failure to rescue rates (in some centres even $0 \%$ !) when compared to lower volume centres. Thus, the increased mortality in older patients was attributed to worse preoperative selection and higher failure to rescue rates in patients in the older group. The three most common causes of failure to rescue were: postoperative pneumonia, cardiovascular accidents and postoperative bleeding [21]. Therefore, early recognition and timely management of complications are crucial as regards decreasing mortality in older patients.

Specific surgical complications, such as postoperative pancreatic fistula, delayed gastric emptying, postpancreatectomy hemorrhaging and intraabdominal abscess were comparable between the older and younger group [22]. Barabas et al. concluded their study with an observation that older patients who can successfully complete the course of neoadjuvant therapy and tolerate its associated morbidity probably had adequate physiological reserve to withstand the surgery [23].

When it comes to the overall survival of older patients after pancreatic resection, it was shorter than younger patients. Finlayson observed the 5-year survival of patients following surgery for PC and demonstrated a decrease from $16.4 \%$ in patients aged $65-69$ years to $15.6 \%$ in patients aged $70-79$ years and $11.3 \%$ in patients aged 80 years or older. However, this difference did not achieve statistical significance. Moreover, patients with more than two comorbidities had a 5-year-survival rate of $10 \%$ compared with $14 \%$ in patients with fewer than two comorbidities; the difference was insignificant [24]. This was mainly because older patients did not receive standard treatment for pancreatic cancer. Older patients were less likely to receive a pancreatectomy with concomitant venous resection, achieve negative margins after surgical resection and receive adjuvant chemotherapy treatment. Moreover, older patients might have been excluded, or might have refused standard "aggressive" therapies, which in turn may have affected their long-term survival outcomes [25].

The limitations of the systematic review were retrospective design of the studies, patients with unresectable tumors or those who declined or have been declined surgical treatment leading to potential selection and information bias. Furthermore, the statistical power of this study was not high.

To conclude, pancreatic resection due to cancer can be performed safely in older patients with acceptable risk in high-volume centres where operations were performed by experienced surgeons. Chronological age alone should not be the only determinant for the selection of patients for surgical treatment. In fit and pre-frail patients, aggressive surgery is recommended to achieve clear surgical margins. However, these data have to be confirmed in large prospective studies with the consideration of non-operative treatment, particularly, when biological age is taken into consideration.

\section{Minimal invasive surgery in pancreatic cancer}

In 2021, Zhu et al. performed a systematic review and metanalysis of seven retrospective studies involving 2727 patients. Three of them compared a minimal invasive pancreaticoduodenectomy (MIP) and an open pancreaticoduodenectomy (OPD) in older patients, two compared MIP between older and young patients and two included both outcomes. Compared to those with OPD, older patients who underwent minimal invasive surgery had a lower 90 -day mortality (OR 0.56; $95 \%$ Cl: $0.32-0.97 ; p=0.04)$ and less delayed gastric emptying (OR 0.54; 95\% Cl: 0.33-0.88; $p=0.01$ ). On the other hand, no significant difference was observed in terms of 30-day mortality, major morbidity, postoperative pancreatic fistula (grade B/C), postoperative hemorrhaging, reoperations, 30day readmissions and operative time. There was no significant difference in operative time between MIP and OPD after the learning curve in case of early cancer stage [26]. However, we have to be aware that most of the studies included in the metanalysis had significant selection bias regarding who was a candidate for minimal invasive surgery; excluding those patients with larger tumours, vascular involvement and prior surgery. Most of the studies were underpowered. Long-term outcomes, such as overall survival and disease-free survival, were not systematically reported $[27,28]$.

To conclude, this meta-analysis demonstrates that MIPD is a safe and feasible procedure for select older patients if performed by experienced surgeons from high-volume pancreatic surgery centres. Older patients can benefit from the advantages of minimal invasive surgery in the case of uneventful postoperative course [29].

\section{Quality of life after PD}

All studies showed a deterioration in patient-reported outcomes and functions after a pancreatoduodenectomy. They were at their worst level after 6 weeks after the resection. Most of the symptoms abated after 3 months and function after 6 months when adjuvant chemotherapy was not introduced. Quality of life has been shown to recover 12 months from potentially curative surgery [30]. In turn, body weight, triceps skin-fold thickness and serum albumin levels recovered in the following 3-6 months [31, 32]. In 33-55\% of all studied patients with $\mathrm{PC}$, depression was observed; this was significantly higher than in patients with other malignancies [33]. Diouf et al. identified fatigue, appetite loss and functioning as the most important aspects of quality of life in predicting prognosis [34] 
In the long-term evaluation, the quality of life of patients who had remained recurrence-free following surgery for PC, was generally good within 24 months of follow-up. Between $24 \%$ and $69 \%$ of patients may develop fat-soluble vitamin and mineral deficiencies, dumping syndrome, diabetes mellitus and delayed gastric emptying [35].

\section{Adjuvant / neoadjuvant treatment}

There is still an under representation of older patients in clinical trials evaluating the role of perioperative treatment. Therefore, it is impossible to draw evidence-based conclusions on the optimal treatment model, not to mention the treatment of frail patients [36]. Adjuvant therapy includes systemic chemotherapy to reduce the risk of distant metastases (80\% of cases) and chemoradiotherapy to reduce the risk of locoregional failure (20\% of cases). European guidelines favour chemotherapy alone and do not recommend the use of chemoradiotherapy outside of a clinical trial setting. In the United States, guidelines recommend chemoradiotherapy as a suitable alternative to adjuvant chemotherapy alone [37].

Based on mainly retrospective studies, it can be concluded that despite the repeatedly demonstrated benefits of adjuvant treatment (increasing 5-year overall survival by up to $25 \%$, independently of age), multimodal therapy seems to be underutilised in the older population [38]. Parmar et al. showed that only $11 \%$ of over 10 thousand studied patients older than 65 years with PC received surgery and chemotherapy. Taking into consideration the whole population, less than half of patients undergoing resection received chemotherapy [39]. In the older group, this could be due to longer postoperative recovery and the subjective perception of the limited life expectancy of patients with $\mathrm{PC}$, considering mainly the chronological age, the ASA or ECOG/WHO scale. However, available data shows that older patients may benefit from chemotherapy in both adjuvant and advanced disease settings. Despite the discordant results, gemcitabine-based treatment and dose-adapted fluorouracil combination regimens seem to be effective and well tolerated in this subset of patients [40]. Not receiving chemotherapy was an independent prognostic factor for poor
OS in the older population (HR: 1.89; Cl: 1.27-2.78; $\mathrm{p}=0.002$ ). Patients at the age of $\geq 70$ years of age who received adjuvant treatment had a survival benefit of the same magnitude as younger patients (21.8 vs. 22.5 months) [41].

Neoadjuvant therapies have been introduced with the aim of downstaging the tumour in order to improve microscopic resection rates. Older patients, with borderline or resectable pancreatic cancer in which the initiation of adjuvant chemotherapy is frequently delayed due to surgical complications, comorbidities and general health status could particularly benefit from this approach. Preoperative therapies also provide a time window allowing not only a clear view of the "ugly" biology of the cancer but also a chance to carry out the multimodal prehabilitation of pre- and frail patients. Barabas et al. observed that older patients who can successfully complete a course of neoadjuvant therapy and tolerate its associated morbidity probably had adequate physiological reserve to withstand surgery. In turn, Miura et al. reported the outcomes associated with neoadjuvant therapy (chemotherapy or chemoradiotherapy) in older patients with resectable or borderline resectable pancreatic cancer. The authors showed that the $75+$ group compared with the younger group had more hospitalisations during the therapy (50 vs. 28\%) and were also less likely to complete the therapy (72.4 vs. 89.5\%). However, among the patients who completed the therapy, there were no significant differences in complication rates or median overall survival between the two groups. In turn, Marmor et al. showed that, compared with chemotherapy, surgical resection is associated with a very small survival advantage in older patients (aged $\geq 80$ years with lymph node metastasis) [42].

In conclusion, the neoadjuvant approach seems to be an attractive treatment option in older patients with borderline resectable pancreatic cancer and for patients who are not candidates for surgery, allowing also for prerehabilitation and reevaluation possibilities. The role of adjuvant therapy has been demonstrated to be beneficial, however, older patients are often not included due to longer postoperative recovery and subjective evaluation of the patient's health status or life expectancy. We urgently need well-designed

Table I. Therapeutic options for pancreatic cancer depending on the cancer stage, including options for frail older patients

\begin{tabular}{|lll}
\hline Stage factors & Fit patients & Frail patients \\
& & \\
\hline $\begin{array}{l}\text { resectable pancreatic } \\
\text { cancer }\end{array}$ & surgery $\rightarrow$ adjuvant chemotherapy & - prehabilitation + reevaluation $\rightarrow$ surgery \\
& & neoadjuvant chemotherapy (in the meantime $+/-$ prehabilitation) $\rightarrow$ \\
& surgery
\end{tabular}


prospective studies evaluating their role in the treatment of the older population with PC. However, the basis for the selection of patients must be biological and not chronological age [43-45].

\section{Palliative treatment}

There are only few studies dedicated to older patients. In the prospective PRODIGE clinical trial, age was an adverse prognostic factor in metastatic PC [46]. In other studies, the use of systemic therapy was proven to have a survival benefit in selected old and very old patients [47]. Considering the results of published studies, Higuera et al. proposed the following treatment for older patients with metastatic PC [48]:

1. Patients $<75$ years old:

- ECOG 0-1: FOLFIRINOX or gemcitabine-nab-paclitaxel schedule,

- ECOG 2: nab-paclitaxel-gemcitabine,

- ECOG 2 or more: best supportive care.

2. Patients $>75$ years old:

- ECOG 0-2: gemcitabine-nab-paclitaxel schedule,

- $\quad$ ECOG $>2$ : best supportive care.

\section{Best supportive care}

At the time of diagnosis, the majority of patients had a locally advanced PC or metastatic disease characterised by a high symptom burden. The most common complications observed in patients with PC are: cachexia (80\%; due to complex pathophysiological processes), pain (75\%; due to pancreatic and celiac plexus infiltration), biliary obstruction (70\%; in case of head location), duodenal obstruction (20\%), and thromboembolic events.

Cachexia is characterised by the loss of skeletal muscle mass that cannot be fully reversed via conventional nutritional support and leads to progressive functional impairments. This state is therefore particularly dangerous for older patients, very often influencing the decision regarding further treatment. Weight stabilisation in patients with PDAC has been associated with improved OS and quality of life [49].

Older patients usually underreport pain. Thus, it remains not poorly treated, leading to a decrease in the quality of life, depression and a deterioration of performance status [50]. Biliary obstruction can be treated successfully with an endoscopically placed stent. However, in the case of a plastic stent, older age was found to be an unfavourable prognostic factor for stent patency [51]. In the case of duodenal obstruction, stent placement or palliative surgery will resolve the symptoms [52].

\section{Conclusions}

Chronological age alone should not be a contraindication for multimodal radical treatment in older patients. The frailty (a surrogate of biological age) evaluation should be the basis for a discussion on treatment planning. At present, it is one of the most reliable factors in older patients.

Therefore, before treatment begins, the following questions should be discussed:

- Is the currently planned treatment strategy correct? Are there any alternative treatment options?

- What is the result of the Comprehensive Geriatric Assessment? Can frailty syndrome be diagnosed in the patient?

- What are the risk of complications?

- What would be the patient's lifespan without treatment?

- What are the goals, preferences and expectations of the patient? What effect might treatment have on these goals?

- Is it possible to improve the patient's state prior to the surgical procedure?

Surgical resection is the only curative treatment option for PC and pancreas surgery has become a safe procedure for older patients in high-volume hospitals when operated on by an experienced surgeon. Fit and pre-frail (after prehabilitation) patients, according to the Comprehensive Geriatric Assessment, should be qualified for the same treatment as younger patients. Frail patients should be discussed in an oncogeriatric meeting. We do not have good data to draw a conclusion regarding what would be the most beneficial for this subpopulation of older patients both in the short- and long-term follow-up. In the case of severe frailty, best supportive treatment can be the best option.

The goal of the modifications should be a reduction in surgical stress, since in older patients, the pathological outcome and postoperative complications are the most important predictors of survival. Therefore, preoperative CGA in older patients is not the end of geriatric intervention, but merely the beginning.

Conflict of interest: none declared

\author{
Jakub Kenig \\ Jagiellonian University Medical College \\ I Chair of General Surgery \\ Department of General, Oncologic, Gastrointestinal Surgery and \\ Transplantology \\ ul. Jakubowskiego 2 \\ 30-688 Kraków, Poland \\ e-mail:jkenig@cm-uj.krakow.pl
}

Received: 30 Aug 2021

Accepted: 14 Sep 2021

\section{References}

1. Smith BD, Smith GL, Hurria A, et al. Future of cancer incidence in the United States: burdens upon an aging, changing nation. J Clin Oncol. 2009; 27(17): 2758-2765, doi: $10.1200 / J C O .2008 .20 .8983$, indexed in Pubmed: 19403886.

2. Rahib L, Smith B, Aizenberg R, et al. Projecting Cancer Incidence and Deaths to 2030: The Unexpected Burden of Thyroid, Liver, and Pancreas Cancers in the United States. Cancer Research. 2014; 74(11):2913-2921, doi: 10.1158/0008-5472.can-14-0155. 
3. Amin S, Lucas A, Frucht H. Evidence for Treatment and Survival Disparities by Age in Pancreatic Adenocarcinoma. Pancreas. 2013; 42(2): 249-253, doi: 10.1097/mpa.0b013e31825f3af4.

4. Puts MTE, Santos B, Hardt J, et al. An update on a systematic review of the use of geriatric assessment for older adults in oncology. Ann Oncol. 2014; 25(2): 307-315, doi: 10.1093/annonc/mdt386, indexed in Pubmed: 24256847.

5. Kamisawa T, Yuyang T, Egawa N, et al. Characteristics of pancreatic carcinoma in the elderly. Int J Pancreatol. 1998; 24(1): 31-34, doi: 10.1007/ BF02787528, indexed in Pubmed: 9746887.

6. Kenig J. Oncogeriatrics (part 1.). Frailty in older adults with cancer. Nowotwory. Journal of Oncology. 2019; 69(2): 55-57, doi: 10.5603/ njo.2019.0010.

7. Loh KP, Soto-Perez-de-Celis E, Hsu T, et al. What Every Oncologist Should Know About Geriatric Assessment for Older Patients With Cancer: Young International Society of Geriatric Oncology Position Paper. J Oncol Pract. 2018; 14(2): 85-94, doi: 10.1200/JOP.2017.026435, indexed in Pubmed: 29436306.

8. Klabunde $\mathrm{CN}$, Ambs A, Keating NL, et al. The role of primary care physicians in cancer care. J Gen Intern Med. 2009; 24(9): 1029-1036, doi: 10.1007/s11606-009-1058-x, indexed in Pubmed: 19597893.

9. Grodzicki T, Kenig J. Problemy okołooperacyjne u osób w wieku podeszłym. PZWL Wydawnictwo Lekarskie 2018.

10. Kenig J, Szabat K. Oncogeriatrics (part 7.). Geriatric assessment for older patients with cancer. Nowotwory. Journal of Oncology. 2020; 70(4): 153-157, doi: 10.5603/njo.2020.0031.

11. Rostoft $S$, van Leeuwen B. Frailty assessment tools and geriatric assessment in older patients with hepatobiliary and pancreatic malignancies. Eur J Surg Oncol. 2021; 47(3 Pt A): 514-518, doi: 10.1016/j. ejso.2020.08.024, indexed in Pubmed: 32933803

12. He W, Zhao H, Chan W, et al. Underuse of surgical resection among elderly patients with early-stage pancreatic cancer. Surgery. 2015; 158(5): 1226-1234, doi: 10.1016/j.surg.2015.04.031, indexed in Pubmed: 26138347.

13. Park HM, Park SJ, Han SS, et al. Surgery for elderly patients with resectable pancreatic cancer, a comparison with non-surgical treatments: a retrospective study outcomes of resectable pancreatic cancer. BMC Cancer. 2019; 19(1): 1090, doi: 10.1186/s12885-019-6255-3, indexed in Pubmed: 31718565.

14. Marmor S, Burke EE, Virnig BA, et al. A comparative analysis of survival outcomes between pancreatectomy and chemotherapy for elderly patients with adenocarcinoma of the pancreas. Cancer. 2016; 122(21): 3378-3385, doi: 10.1002/cncr.30199, indexed in Pubmed: 27419382.

15. Baram A, Moaven O, Tan E, et al. Postoperative outcomes in elderly patients undergoing pancreatic resection for pancreatic adenocarcinoma: A systematic review and meta-analysis. Int J Surg. 2019; 72: 59-68, doi: 10.1016/j.jju.2019.09.030, indexed in Pubmed: 31580919.

16. Lee DY, Schwartz JA, Wexelman B, et al. Outcomes of pancreaticoduodenectomy for pancreatic malignancy in octogenarians: an American College of Surgeons National Surgical Quality Improvement Program analysis. Am J Surg. 2014; 207(4): 540-548, doi: 10.1016/j. amjsurg.2013.07.042, indexed in Pubmed: 24560585.

17. Lightner $\mathrm{AM}$, Glasgow $\mathrm{RE}$, Jordan $\mathrm{TH}$, et al. Pancreatic resection in the elderly. J Am Coll Surg. 2004; 198(5): 697-706, doi: 10.1016/j.jamcollsurg.2003.12.023, indexed in Pubmed: 15110802.

18. Bathe $\mathrm{OF}$, Levi $\mathrm{D}, \mathrm{Caldera} \mathrm{H}$, et al. Radical resection of periampullary tumors in the elderly: evaluation of long-term results. World J Surg. 2000; 24(3): 353-358, doi: 10.1007/s002689910056, indexed in Pubmed: 10658072.

19. Casadei R, Ricci C, Lazzarini E, et al. Pancreatic resection in patients 80 years or older: a meta-analysis and systematic review. Pancreas. 2014; 43(8): 1208-1218, doi: 10.1097/MPA.0000000000000182, indexed in Pubmed: 25333405.

20. Mauriello C, Polistena A, Gambardella C, et al. Pancreatic stump closure after pancreatoduodenectomy in elderly patients: a retrospective clinical study. Aging Clin Exp Res. 2017; 29(Suppl 1): 35-40, doi: 10.1007/ s40520-016-0657-8, indexed in Pubmed: 27837458.

21. Tamirisa NP, Parmar AD, Vargas GM, et al. Relative Contributions of Complications and Failure to Rescue on Mortality in Older Patients Undergoing Pancreatectomy. Ann Surg. 2016; 263(2): 385-391, doi: 10.1097/SLA.0000000000001093, indexed in Pubmed: 25563871.

22. Finlayson E, Fan Z, Birkmeyer JD. Outcomes in octogenarians undergoing high-risk cancer operation: a national study. J Am Coll Surg. 2007; 205(6): 729-734, doi: 10.1016/j.jamcollsurg.2007.06.307, indexed in Pubmed: 18035254

23. Hodul P, Tansey J, Golts E, et al. Age is not a contraindication to pancreaticoduodenectomy. Am Surg. 2001; 67: 270-275.
24. Ito Y, Kenmochi T, Irino T, et al. The impact of surgical outcome after pancreaticoduodenectomy in elderly patients. World J Surg Oncol. 2011; 9: 102, doi: 10.1186/1477-7819-9-102, indexed in Pubmed: 21906398.

25. Bathe OF, Levi $\mathrm{D}$, Caldera $\mathrm{H}$, et al. Radical resection of periampullary tumors in the elderly: evaluation of long-term results. World J Surg. 2000; 24(3): 353-358, doi: 10.1007/s002689910056, indexed in Pubmed: 10658072.

26. Delitto D, Luckhurst CM, Black BS, et al. Oncologic and Perioperative Outcomes Following Selective Application of Laparoscopic Pancreaticoduodenectomy for Periampullary Malignancies. J Gastrointest Surg. 2016; 20(7): 1343-1349, doi: 10.1007/s11605-016-3136-9, indexed in Pubmed: 27142633.

27. Chapman BC, Gajdos C, Hosokawa P, et al. Comparison of laparoscopic to open pancreaticoduodenectomy in elderly patients with pancreatic adenocarcinoma. Surg Endosc. 2018; 32(5): 2239-2248, doi: 10.1007/ s00464-017-5915-0, indexed in Pubmed: 29067580.

28. Meng L, Xia Q, Cai Y, et al. Impact of Patient Age on Morbidity and Survival Following Laparoscopic Pancreaticoduodenectomy. Surg Laparosc Endosc Percutan Tech. 2019; 29(5): 378-382, doi: 10.1097/ SLE.0000000000000670, indexed in Pubmed: 31107854.

29. Zhu J, Wang G, Du P, et al. Minimally Invasive Pancreaticoduodenectomy in Elderly Patients: Systematic Review and Meta-Analysis. World J Surg. 2021; 45(4): 1186-1201, doi: 10.1007/s00268-020-05945-w, indexed in Pubmed: 33458781.

30. Arvaniti M, Danias N, Theodosopoulou E, et al. Quality of Life Variables Assessment, Before and After Pancreatoduodenectomy (PD): Prospective Study. Glob J Health Sci. 2015; 8(6): 203-210, doi: 10.5539/gjhs. v8n6p203, indexed in Pubmed: 26755486.

31. Park JW, Jang JY, Kim EJ, et al. Effects of pancreatectomy on nutritional state, pancreatic function and quality of life. Br J Surg. 2013; 100(8): 1064-1070, doi: 10.1002/bjs.9146, indexed in Pubmed: 23616030.

32. Lindkvist B, Phillips ME, Domínguez-Muñoz JE. Clinical, anthropometric and laboratory nutritional markers of pancreatic exocrine insufficiency: Prevalence and diagnostic use. Pancreatology. 2015; 15(6): 589-597, doi: 10.1016/j.pan.2015.07.001, indexed in Pubmed: 26243045.

33. Bye A, Jordhøy MS, Skjegstad G, et al. Symptoms in advanced pancreatic cancer are of importance for energy intake. Support Care Cancer. 2013; 21(1): 219-227, doi: 10.1007/s00520-012-1514-8, indexed in Pubmed: 22684989.

34. Diouf $M$, Filleron T, Pointet $A L$, et al. Prognostic value of health-related quality of life in patients with metastatic pancreatic adenocarcinoma: a random forest methodology. Qual Life Res. 2016; 25(7): 1713-1723, doi: 10.1007/s11136-015-1198-x, indexed in Pubmed: 26615615.

35. Cloyd JM, Tran Cao HS, Petzel MQB, et al. Impact of pancreatectomy on long-term patient-reported symptoms and quality of life in recurrence-free survivors of pancreatic and periampullary neoplasms. J Surg Oncol. 2017; 115(2): 144-150, doi: 10.1002/jso.24499, indexed in Pubmed: 27859270.

36. Scher KS, Hurria A. Under-representation of older adults in cancer registration trials: known problem, little progress. J Clin Oncol. 2012; 30(17): 2036-2038, doi: 10.1200/JCO.2012.41.6727, indexed in Pubmed: 22547597.

37. European Society of Medical Oncology. Cancer of the pancreas: ESMO Clinical Practice Guidelines for diagnosis, treatment and follow-up. 2015. http://www.esmo.org/Guidelines/Gastrointestinal-Cancers/ Cancer-of-the-Pancreas.

38. National Comprehensive Cancer Network. NCCN Clinical Practice Guidelines Pancreatic Adenocarcinoma (Version 2.2017). https://www. nccn.org/professionals/physician_gls/f_guidelines.asp\#pancreatic.

39. Higuera $\mathrm{O}$, Ghanem I, Nasimi R, et al. Management of pancreatic cancer in the elderly. World J Gastroenterol. 2016; 22(2): 764-775, doi: 10.3748/ wjg.v22.i2.764, indexed in Pubmed: 26811623.

40. Parmar AD, Vargas GM, Tamirisa NP, et al. Trajectory of care and use of multimodality therapy in older patients with pancreatic adenocarcinoma. Surgery. 2014; 156(2): 280-289, doi: 10.1016/j.surg.2014.03.001, indexed in Pubmed: 24851723.

41. Macchini M, Chiaravalli M, Zanon S, et al. Chemotherapy in elderly patients with pancreatic cancer: Efficacy, feasibility and future perspectives. Cancer Treat Rev. 2019; 72: 1-6, doi: 10.1016/j.ctrv.2018.10.013, indexed in Pubmed: 30414985.

42. Nagrial AM, Chang DK, Nguyen NQ, et al. Australian Pancreatic Cancer Genome Initiative. Adjuvant chemotherapy in elderly patients with pancreatic cancer. Br J Cancer. 2014; 110(2): 313-319, doi: 10.1038/ bjc.2013.722, indexed in Pubmed: 24263063.

43. Marmor S, Burke EE, Virnig BA, et al. A comparative analysis of survival outcomes between pancreatectomy and chemotherapy for elderly 
patients with adenocarcinoma of the pancreas. Cancer. 2016; 122(21): 3378-3385, doi: 10.1002/cncr.30199, indexed in Pubmed: 27419382.

44. Lim KH, Chung E, Khan A, et al. Neoadjuvant therapy of pancreatic cancer: the emerging paradigm? Oncologist. 2012; 17(2): 192-200, doi: 10.1634/theoncologist.2011-0268, indexed in Pubmed: 22250057.

45. Liao WC, Chien KL, Lin YL, et al. Adjuvant treatments for resected pancreatic adenocarcinoma: a systematic review and network meta-analysis. Lancet Oncol. 2013; 14(11): 1095-1103, doi: 10.1016/S14702045(13)70388-7, indexed in Pubmed: 24035532.

46. Miura J, Krepline A, Duelge $K_{1}$ et al. Neoadjuvant therapy for pancreatic cancer in patients older than age 75. J Clin Oncol. 2014; 32(3_suppl): 287-287, doi: 10.1200/jco.2014.32.3_suppl.287.

47. Conroy T, Desseigne F, Ychou M, et al. Groupe Tumeurs Digestives of Unicancer, PRODIGE Intergroup. FOLFIRINOX versus gemcitabine for metastatic pancreatic cancer. N Engl J Med. 2011; 364(19): 1817-1825, doi: 10.1056/NEJMoa1011923, indexed in Pubmed: 21561347.

48. Aldoss I, Tashi T, Gonsalves W, et al. Role of chemotherapy in the very elderly patients with metastatic pancreatic cancer - A Veterans Affa- irs Cancer Registry analysis. J Geriatr Oncol. 2011; 2(3): 209-214, doi: 10.1016/j.jgo.2011.02.003.

49. Higuera O, Ghanem I, Nasimi R, et al. Management of pancreatic cance in the elderly. World J Gastroenterol. 2016; 22(2): 764-775, doi: 10.3748/ wjg.v22.i2.764, indexed in Pubmed: 26811623.

50. Tuca A, Jimenez-Fonseca P, Gascón P.Clinical evaluation and optimal management of cancer cachexia. Crit Rev Oncol Hematol. 2013; 88(3): 625-636, doi: 10.1016/j.critrevonc.2013.07.015, indexed in Pubmed: 23953794.

51. Torgerson S, Wiebe LA. Supportive care of the patient with advanced pancreatic cancer. Oncology (Williston Park). 2013; 27(3): 183-190, indexed in Pubmed: 23687787.

52. Matsuda Y, Shimakura K, AkamatsuT. Factors affecting the patency of stents in malignant biliary obstructive disease: univariate and multivariate analysis. Am J Gastroenterol. 1991; 86(7): 843-849, indexed in Pubmed: 2058626.

53. Jeurnink SM, van Eijck CHJ, Steyerberg EW, et al. Stent versus gastrojejunostomy for the palliation of gastric outlet obstruction: a systematic review. BMC Gastroenterol. 2007; 7: 18, doi: 10.1186/1471-230X-7-18, indexed in Pubmed: 17559659. 